\title{
On some classes of solvable systems of difference equations
}

\author{
Stevo Stević ${ }^{1,2,3,4^{*}}$, Bratislav Iričanin ${ }^{5}$, Witold Kosmala ${ }^{6}$ and Zdeněk Šmarda ${ }^{4}$
}

\author{
"Correspondence: sstevic@ptt.rs \\ ${ }^{1}$ Mathematical Institute of the \\ Serbian Academy of Sciences, \\ Beograd, Serbia \\ ${ }^{2}$ Department of Medical Research, \\ China Medical University Hospital, \\ China Medical University, Taichung, \\ Taiwan, Republic of China \\ Full list of author information is \\ available at the end of the article
}

\begin{abstract}
In a recent paper several periodic systems of difference equations have been presented. We show in an elegant way that all the results therein follow from some known ones. We also show how some extensions of the systems of difference equations can be solved.
\end{abstract}

MSC: 39A05; 39A06

Keywords: System of difference equations; Periodicity; General solution; Difference equation with interlacing indices

\section{Introduction}

Here we use the following standard notations: $\mathbb{N}$ stands for the set of all natural numbers, $\mathbb{Z}$ stands for the set of all integers, for an $l \in \mathbb{Z}, \mathbb{N}_{l}$ is defined as $\{n \in \mathbb{Z}: n \geq l\}, \mathbb{R}$ denotes the set of all real numbers, and $\mathbb{C}$ denotes the set of all complex numbers. Let $k, l \in \mathbb{Z}$, $k \leq l$, then the notation $j=\overline{k, l}$ denotes the set of all $j \in \mathbb{Z}$ such that $k \leq j \leq l$.

Solvability of difference equations has been studied for a long time. Basic classes of solvable difference equations and systems were found during the eighteenth century, and since that time the books which contain results on the topic have more or less presented the old original methods or their modifications and refinements which were obtained during the nineteenth century (see, e.g., [1-11]). For some applications of solvability methods of difference equations, see, e.g., [1, 3-5, 8, 10-19].

Since theory of solvability of difference equations has not given new important or effective general methods for investigating solutions to the equations, the study turned to some other directions, such us finding asymptotic behavior or growth rate of their solutions. Some combinations of these methods with solvability methods for difference equations can be found, for example, in the study of the long-term behavior of solutions to the bilinear difference equation

$$
x_{n+1}=\frac{a x_{n}+b}{c x_{n}+d}, \quad n \in \mathbb{N}_{0} \text {, }
$$

where parameters $a, b, c, d$ and the initial value $x_{0}$ are real or complex numbers (see, e.g., $[1,2,12,13,15,20,21])$, which is a basic example of solvable nonlinear difference equation. Its extension with nonconstant coefficients is an equation of a huge interest since it is equivalent to the homogeneous linear difference equation of second-order. How equation

(c) The Author(s) 2019. This article is distributed under the terms of the Creative Commons Attribution 4.0 International License (http://creativecommons.org/licenses/by/4.0/), which permits unrestricted use, distribution, and reproduction in any medium, provided you give appropriate credit to the original author(s) and the source, provide a link to the Creative Commons license, and indicate if changes were made. 
(1) is solved can be found, e.g., in [2, 5, 10, 15, 21, 22]. For some recent results on representations of solutions to equation (1) in terms of Fibonacci-type sequences, see [23, 24], and the references therein. Some basics on Fibonacci sequences can be found, e.g., in [25] and [26].

We are witnesses that the area of solvability of difference equations and related ones reattracts some attention. One of the reasons for the recent interest is the use of computer algebra systems, which can help in finding or guessing closed-form formulas for solutions to some difference equations and systems. The computer algebra systems are certainly useful, but in the majority cases the authors obtain results which are known or easily follow from the known ones (see, e.g., some of our comments in [23, 24, 27-29] related to the issue).

Our renewed interest in the topic began in 2004 when S. Stević studied the following difference equation:

$$
x_{n+1}=\frac{x_{n-1}}{a+b x_{n-1} x_{n}}, \quad n \in \mathbb{N}_{0},
$$

and gave a theoretical explanation for solvability of the equation. Continuation of the study can be found, e.g., in [30-35]. Related methods and ideas have been later used and developed in many papers (see, e.g., [27-29, 36] and the references therein). The main idea in the papers is to employ some changes of variables which would reduce the equations/systems studied therein to some well-known solvable ones (quite frequently it is the linear first-order difference equation).

Another area of recent interest is concrete systems of difference equations, with a special interest in symmetric and close-to-symmetric ones. The study of such systems was essentially initiated and popularized by Papaschinopoulos and Schinas (see, e.g., [37-44]). Many of our papers are also devoted to the area (see, e.g., [17, 23, 24, 27, 29, 32, 33, 36] and the references therein). For other related results, including the ones on invariants of difference equations, see, e.g., [39, 40, 43, 45, 46] and the references therein. Let us also mention that some more complex solvable difference equations and systems can be found, e.g., in $[17,33,47-51]$, but the idea is essentially the same as in the above-mentioned papers, that is, some connections of studied difference equations and systems to some solvable ones are found.

A vector sequence $\left(\vec{x}_{n}\right)_{n \in \mathbb{N}_{l}}=\left(x_{n}^{(1)}, \ldots, x_{n}^{(k)}\right)_{n \in \mathbb{N}_{l}} \in \mathbb{C}^{k}$ is called eventually periodic with period $p \in \mathbb{N}$ if there is $n_{0} \in \mathbb{N}_{l}$ such that

$$
x_{n+p}^{(i)}=x_{n}^{(i)}
$$

for every $n \geq n_{0}$ and $i=\overline{1, k}$ (see, e.g., $\left.[52,53]\right)$. If $p=1$, then the sequence is called eventually constant [54]. If $n_{0}=l$, then such a sequence is called periodic or more precisely p-periodic. Many authors call both, eventually periodic and periodic sequences, simply as periodic.

In recent paper [46] periodicity of well-defined solutions to the following systems of difference equations has been studied:

$$
\begin{aligned}
& x_{n+1}=\frac{x_{n-k} y_{n-(2 k+1)}}{y_{n-(2 k+1)}-y_{n-k}}, \quad y_{n+1}=\frac{y_{n-k} x_{n-(2 k+1)}}{x_{n-(2 k+1)}-x_{n-k}}, \quad n \in \mathbb{N}_{0}, \\
& x_{n+1}=-\frac{x_{n-k} y_{n-(2 k+1)}}{y_{n-(2 k+1)}+y_{n-k}},
\end{aligned}
$$




$$
\begin{array}{lll}
x_{n+1}=\frac{x_{n-k} y_{n-(2 k+1)}}{y_{n-(2 k+1)}+y_{n-k}}, & y_{n+1}=\frac{y_{n-k} x_{n-(2 k+1)}}{-x_{n-(2 k+1)}+x_{n-k}}, & n \in \mathbb{N}_{0}, \\
x_{n+1}=\frac{x_{n-k} y_{n-(2 k+1)}}{-y_{n-(2 k+1)}+y_{n-k}}, & y_{n+1}=\frac{y_{n-k} x_{n-(2 k+1)}}{x_{n-(2 k+1)}+x_{n-k}}, & n \in \mathbb{N}_{0},
\end{array}
$$

where $k \in \mathbb{N}_{0}$.

Note that systems (2)-(5) are special cases of the following system of difference equations:

$$
x_{n+1}=\frac{x_{n-k} y_{n-(2 k+1)}}{a y_{n-(2 k+1)}+b y_{n-k}}, \quad y_{n+1}=\frac{y_{n-k} x_{n-(2 k+1)}}{c x_{n-(2 k+1)}+d x_{n-k}}, \quad n \in \mathbb{N}_{0} \text {, }
$$

where $k \in \mathbb{N}_{0}$, whereas parameters $a, b, c, d$ and initial values $x_{-j}, y_{-j}, j=\overline{0,2 k+1}$, are complex numbers. Namely, system (2) is obtained with $a=-b=c=-d=1$, system (3) is obtained with $a=b=c=d=-1$, system (4) is obtained with $a=b=-c=d=1$, whereas system (5) is obtained with $-a=b=c=d=1$.

We first study system (6) and then turn to the special cases of the system in (2)-(5). We show that all the results in [46] follow from some known ones. We also show how some extensions of the systems of difference equations can be solved.

\section{On system (6) and the results in [46]}

In this section we first study the structural form of system (6), and then by using the analysis we discuss systems (2)-(5).

\subsection{Analysis of the structural form of system (6)}

Here we investigate the structural form of system (6).

Since the initial values of the system are $x_{-j}, y_{-j}, j=\overline{0,2 k+1}$, the first terms which are calculated by the equations in (6) are $x_{1}$ and $y_{1}$. They are obtained for $n=0$. We have

$$
x_{1}=\frac{x_{-k} y_{-(2 k+1)}}{a y_{-(2 k+1)}+b y_{-k}} \quad \text { and } \quad y_{1}=\frac{y_{-k} x_{-(2 k+1)}}{c x_{-(2 k+1)}+d x_{-k}} .
$$

Hence, in calculation of terms $x_{1}$ and $y_{1}$, only terms $x_{-(2 k+1)}, y_{-(2 k+1)}, x_{-k}$, and $y_{-k}$ are used.

Let $n=k+1$. Then from (6) we have

$$
x_{k+2}=\frac{x_{1} y_{-k}}{a y_{-k}+b y_{1}} \quad \text { and } \quad y_{k+2}=\frac{y_{1} x_{-k}}{c x_{-k}+d x_{1}}
$$

From (7) and bearing in mind that $x_{1}$ and $y_{1}$ are calculated by using only terms $x_{-(2 k+1)}$, $y_{-(2 k+1)}, x_{-k}$, and $y_{-k}$, it follows that in calculation of $x_{k+2}$ and $y_{k+2}$ also only terms $x_{-(2 k+1)}$, $y_{-(2 k+1)}, x_{-k}$, and $y_{-k}$ are used.

Let $n=2 k+2$. Then from (6) we have

$$
x_{2 k+3}=\frac{x_{k+2} y_{1}}{a y_{1}+b y_{k+2}} \quad \text { and } \quad y_{2 k+3}=\frac{y_{k+2} x_{1}}{c x_{1}+d x_{k+2}}
$$

From (8) and bearing in mind that $x_{1}, y_{1}, x_{k+2}$, and $y_{k+2}$ are calculated by using only terms $x_{-(2 k+1)}, y_{-(2 k+1)}, x_{-k}$, and $y_{-k}$, it follows that in calculation of $x_{2 k+3}$ and $y_{2 k+3}$ also only terms $x_{-(2 k+1)}, y_{-(2 k+1)}, x_{-k}$, and $y_{-k}$ are used. 
Assume that for some $m \in \mathbb{N}$ we have proved that $x_{j(k+1)+1}, y_{j(k+1)+1},-2 \leq j \leq m$, are calculated by using only terms $x_{-(2 k+1)}, y_{-(2 k+1)}, x_{-k}$, and $y_{-k}$. From the hypothesis and since

$$
\begin{aligned}
x_{(m+1)(k+1)+1} & =\frac{x_{m(k+1)+1} y_{(m-1)(k+1)+1}}{a y_{(m-1)(k+1)+1}+b y_{m(k+1)+1}}, \\
y_{(m+1)(k+1)+1} & =\frac{y_{m(k+1)+1} x_{(m-1)(k+1)+1}}{c x_{(m-1)(k+1)+1}+d x_{m(k+1)+1}},
\end{aligned}
$$

it is easy to see that in calculation of $x_{(m+1)(k+1)+1}$ and $y_{(m+1)(k+1)+1}$ also only terms $x_{-(2 k+1)}$, $y_{-(2 k+1)}, x_{-k}$, and $y_{-k}$ are used.

Hence, by induction we have proved that in calculation of all terms of the sequences $\left(x_{m(k+1)+1}\right)_{m \geq-2}$ and $\left(y_{m(k+1)+1}\right)_{m \geq-2}$ also only terms $x_{-(2 k+1)}, y_{-(2 k+1)}, x_{-k}$, and $y_{-k}$ are used.

The same argument shows that for each fixed $j \in\{1,2, \ldots, k+1\}$, in calculation of all terms of the sequences $\left(x_{m(k+1)+j}\right)_{m \geq-2}$ and $\left(y_{m(k+1)+j}\right)_{m \geq-2}$, only terms $x_{j-(2 k+2)}, y_{j-(2 k+2)}$, $x_{j-(k+1)}$, and $y_{j-(k+1)}$ are used.

Let $S_{j}, j=\overline{1, k+1}$, be the sets defined as follows:

$$
S_{j}:=\{(k+1) m+j: m \geq-2\}, \quad j=\overline{1, k+1} .
$$

It is easy to see that

$$
S_{i} \cap S_{j}=\emptyset, \quad i \neq j,
$$

and

$$
\bigcup_{j=1}^{k+1} S_{j}=\mathbb{N}_{-(2 k+1)}
$$

Equality (10) implies that when $i \neq j$, the sequences $\left(x_{m(k+1)+j}, y_{m(k+1)+j}\right)_{m \geq-2}$ and $\left(x_{m(k+1)+i}\right.$, $\left.y_{m(k+1)+i}\right)_{m \geq-2}$ are independent of each other (i.e., not connected at all) since the sets of indices are disjoint, while (11) means that the union of the sequences $\left(x_{m(k+1)+j}\right)_{m \geq-2}$, $j=\overline{1, k+1}$, forms the whole sequence $\left(x_{n}\right)_{n \geq-(2 k+1)}$, whereas the union of the sequences $\left(y_{m(k+1)+j}\right)_{m \geq-2}, j=\overline{1, k+1}$, forms the whole sequence $\left(y_{n}\right)_{n \geq-(2 k+1)}$. Hence, the sequences $\left(x_{m(k+1)+j}, y_{m(k+1)+j}\right)_{m \geq-2}, j=\overline{1, k+1}$, form a partition of the sequence $\left(x_{n}, y_{n}\right)_{n \geq-(2 k+1)}$.

Let

$$
\begin{aligned}
& x_{m}^{(j)}:=x_{(k+1) m+j}, \\
& y_{m}^{(j)}:=y_{(k+1) m+j},
\end{aligned}
$$

for $m \geq-2$, where $j=\overline{1, k+1}$.

Then system (6) can be written as

$$
x_{m+1}^{(j)}=\frac{x_{m}^{(j)} y_{m-1}^{(j)}}{a y_{m-1}^{(j)}+b y_{m}^{(j)}}, \quad y_{m+1}^{(j)}=\frac{y_{m}^{(j)} x_{m-1}^{(j)}}{c x_{m-1}^{(j)}+d x_{m}^{(j)}}, \quad m \geq-1,
$$

for $j \in\{1, \ldots, k+1\}$. 
From this it follows that the sequences $\left(x_{m}^{(j)}, y_{m}^{(j)}\right)_{m \geq-2}, j=\overline{1, k+1}$, are $k+1$ independent solutions to the following second-order system of difference equations:

$$
\widehat{x}_{m+1}=\frac{\widehat{x}_{m} \widehat{y}_{m-1}}{a \widehat{y}_{m-1}+b \widehat{y}_{m}}, \quad \widehat{y}_{m+1}=\frac{\widehat{y}_{m} \widehat{x}_{m-1}}{c \widehat{x}_{m-1}+d \widehat{x}_{m}}, \quad m \geq-1,
$$

with the initial values $x_{j-(2 k+2)}, y_{j-(2 k+2)}, x_{j-(k+1)}$, and $y_{j-(k+1)}, j=\overline{1, k+1}$, respectively. Hence, system (6) is a system of difference equations with interlacing indices [27]. If such a system/equation is reduced to $l$ systems/equations, which are not all systems/equations with interlacing indices, we say that the system/equation is a system/equation with interlacing indices of order $l$.

It has been recently shown in [29] that system (15) is solvable, where also the long-term behavior of its solutions has been described in detail in many cases. Hence, the long-term behavior of solutions to system (6) practically directly follows from the long-term behavior of solutions to system (15).

\subsection{On the results on systems (2)-(5) quoted in [46]}

Here we discuss in detail the results quoted in [46].

The first result quoted in [46] is the following theorem, which was proved by a long calculatory-inductive argument.

Theorem 1 Let $\left(x_{n}, y_{n}\right)_{n \geq-(2 k+1)}$ be a well-defined solution to system (2). Then the following statements hold.

(i) Sequences $\left(x_{n}\right)_{n \geq-(2 k+1)}$ and $\left(y_{n}\right)_{n \geq-(2 k+1)}$ are periodic with period $6(k+1)$.

(ii) We have

$$
\begin{aligned}
& x_{6(k+1) m+l}= \begin{cases}\frac{x_{l-k-1} y_{l-2 k-2}}{y_{l-2 k-2}-y_{l-k-1}}, & 1 \leq l \leq k+1, \\
\frac{y_{l-3 k-3}\left(-x_{l-3 k-3}+x_{l-2 k-2}\right)}{y_{l-3 k-3}-y_{l-2 k-2}}, & k+2 \leq l \leq 2 k+2, \\
\frac{y_{l-3 k-3}\left(-x_{l-4 k-4}+x_{l-3 k-3}\right)}{y_{l-4 k-4} y_{l-3 k-3}}, & 2 k+3 \leq l \leq 3 k+3, \\
\frac{y_{l-4 k-4} x_{l-5 k-5}}{-y_{l-5 k-5}+y_{l-4 k-4}}, & 3 k+4 \leq l \leq 4 k+4, \\
x_{l-6 k-6}, & 4 k+5 \leq l \leq 6 k+6,\end{cases} \\
& y_{6(k+1) m+l}= \begin{cases}\frac{y_{l-k-1} x_{l-2 k-2}}{x_{l-2 k-2}-x_{l-k-1}}, & 1 \leq l \leq k+1, \\
\frac{x_{l-3 k-3}\left(-y_{l-3 k-3}+y_{l-2 k-2}\right)}{x_{l-3 k-3}-x_{l-2 k-2}}, & k+2 \leq l \leq 2 k+2, \\
\frac{x_{l-3 k-3}\left(-y_{l-4 k-4}+y_{l-3 k-3}\right)}{x_{l-4 k-4}-x_{l-3 k-3}}, & 2 k+3 \leq l \leq 3 k+3, \\
\frac{x_{l-4 k-4 y_{l-5 k-5}}}{-x_{l-5 k-5}+x_{l-4 k-4}}, & 3 k+4 \leq l \leq 4 k+4, \\
y_{l-6 k-6}, & 4 k+5 \leq l \leq 6 k+6,\end{cases}
\end{aligned}
$$

for $m \in \mathbb{N}_{0}$.

In [29, Theorem 4] (see also Example 4 therein for details) it was proved that every welldefined solution to system (15) with $a=-b=c=-d=1$ is six-periodic. Bearing in mind this fact, as well as the above consideration which shows that the sequences defined in (12) and (13) are $k+1$ independent solutions to the system, it immediately follows that for every well-defined solution to system (2) the sequences $\left(x_{n}\right)_{n \geq-(2 k+1)}$ and $\left(y_{n}\right)_{n \geq-(2 k+1)}$ 
are periodic with period $6(k+1)$. So, Theorem $1(i)$ is a very simple consequence of known results.

Part (ii) is a simple consequence of the independence of solutions $\left(x_{m}^{(j)}, y_{m}^{(j)}\right)_{m \geq-2}, j=$ $\overline{1, k+1}$, to the corresponding non-interlacing system (15), and the following equalities, which are verified by simple calculation (they hold for every well-defined solution $\left(\widehat{x}_{m}, \widehat{y}_{m}\right)_{m \geq-2}$ to system (15))

$$
\begin{aligned}
& \widehat{x}_{0}=\frac{\widehat{x}_{-1} \widehat{y}_{-2}}{\widehat{y}_{-2}-\widehat{y}_{-1}}, \\
& \widehat{x}_{1}=-\frac{\widehat{y}_{-2}\left(\widehat{x}_{-2}-\widehat{x}_{-1}\right)}{\widehat{y}_{-2}-\widehat{y}_{-1}}, \\
& \widehat{x}_{2}=-\frac{\widehat{y}_{-1}\left(\widehat{x}_{-2}-\widehat{x}_{-1}\right)}{\widehat{y}_{-2}-\widehat{y}_{-1}}, \\
& \widehat{x}_{3}=-\frac{\widehat{x}_{-2} \widehat{y}_{-1}}{\widehat{y}_{-2}-\widehat{y}_{-1}}, \\
& \widehat{x}_{4}=\widehat{x}_{-2}, \\
& \widehat{x}_{5}=\widehat{x}_{-1},
\end{aligned}
$$

and due to the symmetry of system (15)

$$
\begin{aligned}
& \widehat{y}_{0}=\frac{\widehat{y}_{-1} \widehat{x}_{-2}}{\widehat{x}_{-2}-\widehat{x}_{-1}}, \\
& \widehat{y}_{1}=-\frac{\widehat{x}_{-2}\left(\widehat{y}_{-2}-\widehat{y}_{-1}\right)}{\widehat{x}_{-2}-\widehat{x}_{-1}}, \\
& \widehat{y}_{2}=-\frac{\widehat{x}_{-1}\left(\widehat{y}_{-2}-\widehat{y}_{-1}\right)}{\widehat{x}_{-2}-\widehat{x}_{-1}}, \\
& \widehat{y}_{3}=-\frac{\widehat{y}_{-2} \widehat{x}_{-1}}{\widehat{x}_{-2}-\widehat{x}_{-1}}, \\
& \widehat{y}_{4}=\widehat{y}_{-2}, \\
& \widehat{y}_{5}=\widehat{y}_{-1},
\end{aligned}
$$

where $\widehat{x}_{-2}, \widehat{y}_{-2}, \widehat{x}_{-1}$, and $\widehat{y}_{-1}$ are replaced with the initial values $x_{j-(2 k+2)}, y_{j-(2 k+2)}, x_{j-(k+1)}$, and $y_{j-(k+1)}, j=\overline{1, k+1}$, respectively.

The second result in [46] is the following theorem, for which it was only said that it is proved similarly to Theorem 1 (by an inductive argument).

Theorem 2 Let $\left(x_{n}, y_{n}\right)_{n \geq-(2 k+1)}$ be a well-defined solution to system (3). Then the following statements hold.

(i) Sequences $\left(x_{n}\right)_{n \geq-(2 k+1)}$ and $\left(y_{n}\right)_{n \geq-(2 k+1)}$ are periodic with period $6(k+1)$. 
(ii) We have

$$
\begin{gathered}
x_{6(k+1) m+l}= \begin{cases}-\frac{x_{l-k-1} y_{l-2 k-2}}{y_{l-2 k-2}+y_{l-k-1},}, & 1 \leq l \leq k+1, \\
\frac{y_{l-3 k-3}\left(x_{l-3 k-3}+x_{l-2 k-2}\right)}{y_{l-3 k-3}+y_{l-2 k-2}}, & k+2 \leq l \leq 2 k+2, \\
\frac{y_{l-3 k-3}\left(x_{l-4 k-4}+x_{l-3 k-3}\right)}{y_{l-4 k-4+y_{l-3 k-3}},} & 2 k+3 \leq l \leq 3 k+3, \\
-\frac{y_{l-4 k-4} x_{-5 k-5}}{y_{l-5 k-5}+y_{l-4 k-4}}, & 3 k+4 \leq l \leq 4 k+4, \\
x_{l-6 k-6}, & 4 k+5 \leq l \leq 6 k+6,\end{cases} \\
y_{6(k+1) m+l}= \begin{cases}-\frac{y_{l-k-1} x_{l-2 k-2}}{x_{l-2 k-2}+x_{l-k-1},}, & 1 \leq l \leq k+1, \\
\frac{x_{l-3 k-3}\left(y_{l-3 k-3}+y_{l-2 k-2}\right)}{x_{l-3 k-3}+x_{l-2 k-2}}, & k+2 \leq l \leq 2 k+2, \\
\frac{x_{l-3 k-3}\left(y_{l-4 k-4}+y_{l-3 k-3}\right)}{x_{l-4 k-4}+x_{l-3 k-3}}, & 2 k+3 \leq l \leq 3 k+3, \\
-\frac{x_{l-4 k-4}+5 k-5}{x_{l-5 k-5}+x_{l-4 k-4}}, & 3 k+4 \leq l \leq 4 k+4, \\
y_{l-6 k-6}, & 4 k+5 \leq l \leq 6 k+6,\end{cases}
\end{gathered}
$$

for $m \in \mathbb{N}_{0}$.

Since system (3) is a special case of system (6), then similar to (12)-(15), we see that the system is equivalent to $k+1$ independent systems of the following form:

$$
\widehat{x}_{m+1}=\frac{\widehat{x}_{m} \widehat{y}_{m-1}}{-\widehat{y}_{m-1}-\widehat{y}_{m}}, \quad \widehat{y}_{m+1}=\frac{\widehat{y}_{m} \widehat{x}_{m-1}}{-\widehat{x}_{m-1}-\widehat{x}_{m}}, \quad m \geq-1
$$

By using the change of variables

$$
\widehat{x}_{m}=(-1)^{m} \widetilde{x}_{m}, \quad \widehat{y}_{m}=(-1)^{m} \widetilde{y}_{m}, \quad m \geq-2,
$$

in (28), it easily follows that

$$
\tilde{x}_{m+1}=\frac{\widetilde{x}_{m} \tilde{y}_{m-1}}{\widetilde{y}_{m-1}-\widetilde{y}_{m}}, \quad \tilde{y}_{m+1}=\frac{\tilde{y}_{m} \tilde{x}_{m-1}}{\widetilde{x}_{m-1}-\widetilde{x}_{m}}, \quad m \geq-1 .
$$

Using again Theorem 4 in [29], we see that every well-defined solution to system (30) is six-periodic, from which along with (29) it follows that every well-defined solution to system (28) is six-periodic. Hence, we have that every well-defined solution to system (3) is periodic with period $6(k+1)$. So, Theorem $2(i)$ is also a very simple consequence of known results.

Part (ii) is a simple consequence of the independence of solutions $\left(x_{m}^{(j)}, y_{m}^{(j)}\right)_{m \geq-2}, j=$ $\overline{1, k+1}$, to system (30), and the following equalities for terms $\widehat{x}_{j}, \widehat{y}_{j}, j=\overline{0,5}$, where $\widehat{x}_{-2}$, $\widehat{y}_{-2}, \widehat{x}_{-1}$, and $\widehat{y}_{-1}$ are replaced with the initial values $x_{j-(2 k+2)}, y_{j-(2 k+2)}, x_{j-(k+1)}$, and $y_{j-(k+1)}$, $j=\overline{1, k+1}$, respectively. Note that due to (29) and (16)-(21), it is not necessary to calculate $\widehat{x}_{j}, j=\overline{0,5}$, directly. Namely, by using the equalities, we have

$$
\begin{aligned}
& \widehat{x}_{0}=\widetilde{x}_{0}=\frac{\widetilde{x}_{-1} \widetilde{y}_{-2}}{\widetilde{y}_{-2}-\widetilde{y}_{-1}}=-\frac{\widehat{x}_{-1} \widehat{y}_{-2}}{\widehat{y}_{-2}+\widehat{y}_{-1}}, \\
& \widehat{x}_{1}=-\widetilde{x}_{1}=\frac{\widetilde{y}_{-2}\left(\widetilde{x}_{-2}-\widetilde{x}_{-1}\right)}{\widetilde{y}_{-2}-\widetilde{y}_{-1}}=\frac{\widehat{y}_{-2}\left(\widehat{x}_{-2}+\widehat{x}_{-1}\right)}{\widehat{y}_{-2}+\widehat{y}_{-1}},
\end{aligned}
$$




$$
\begin{aligned}
& \widehat{x}_{2}=\widetilde{x}_{2}=-\frac{\widetilde{y}_{-1}\left(\widetilde{x}_{-2}-\widetilde{x}_{-1}\right)}{\tilde{y}_{-2}-\widetilde{y}_{-1}}=\frac{\widehat{y}_{-1}\left(\widehat{x}_{-2}+\widehat{x}_{-1}\right)}{\widehat{y}_{-2}+\widehat{y}_{-1}}, \\
& \widehat{x}_{3}=-\widetilde{x}_{3}=\frac{\widetilde{x}_{-2} \widetilde{y}_{-1}}{\widetilde{y}_{-2}-\widetilde{y}_{-1}}=-\frac{\widehat{x}_{-2} \widehat{y}_{-1}}{\widehat{y}_{-2}+\widehat{y}_{-1}}, \\
& \widehat{x}_{4}=\widetilde{x}_{4}=\widetilde{x}_{-2}=\widehat{x}_{-2}, \\
& \widehat{x}_{5}=-\widetilde{x}_{5}=-\widetilde{x}_{-1}=\widehat{x}_{-1} .
\end{aligned}
$$

Since system (28) is symmetric, from (31)-(36) we directly obtain

$$
\begin{aligned}
& \widehat{y}_{0}=-\frac{\widehat{y}_{-1} \widehat{x}_{-2}}{\widehat{x}_{-2}+\widehat{x}_{-1}}, \\
& \widehat{y}_{1}=\frac{\widehat{x}_{-2}\left(\widehat{y}_{-2}+\widehat{y}_{-1}\right)}{\widehat{x}_{-2}+\widehat{x}_{-1}}, \\
& \widehat{y}_{2}=\frac{\widehat{x}_{-1}\left(\widehat{y}_{-2}+\widehat{y}_{-1}\right)}{\widehat{x}_{-2}+\widehat{x}_{-1}}, \\
& \widehat{y}_{3}=-\frac{\widehat{y}_{-2} \widehat{x}_{-1}}{\widehat{x}_{-2}+\widehat{x}_{-1}}, \\
& \widehat{y}_{4}=\widehat{y}_{-2}, \\
& \widehat{y}_{5}=\widehat{y}_{-1} .
\end{aligned}
$$

The next two results quoted in [46, Corollary 4.1] are the following theorems, for which it was also only said that they can be proved similarly to Theorem 1.

Theorem 3 Let $\left(x_{n}, y_{n}\right)_{n \geq-(2 k+1)}$ be a well-defined solution to system (4). Then the following statements hold.

(i) Sequences $\left(x_{n}\right)_{n \geq-(2 k+1)}$ and $\left(y_{n}\right)_{n \geq-(2 k+1)}$ are periodic with period $6(k+1)$.

(ii) We have

$$
\begin{gathered}
x_{6(k+1) m+l}= \begin{cases}\frac{x_{l-k-1} y_{l-2 k-2}}{y_{l-2 k-2}+y_{l-k-1}}, & 1 \leq l \leq k+1, \\
\frac{y_{l-3 k-3}\left(-x_{l-3 k-3}+x_{l-2 k-2}\right)}{y_{l-3 k-3}+y_{l-2 k-2}}, & k+2 \leq l \leq 2 k+2, \\
\frac{y_{l-3 k-3}\left(x_{l-4 k-4}-x_{l-3 k-3}\right)}{y_{l-4 k-4}+y_{l-3 k-3}}, & 2 k+3 \leq l \leq 3 k+3, \\
\frac{y_{l-4 k-4} x_{l-5 k-5}}{y_{l-5 k-5}+y_{l-4 k-4}}, & 3 k+4 \leq l \leq 4 k+4, \\
x_{l-6 k-6}, & 4 k+5 \leq l \leq 6 k+6,\end{cases} \\
y_{6(k+1) m+l}= \begin{cases}\frac{y_{l-k-1} x_{l-2 k-2}}{-x_{l-2 k-2}+x_{l-k-1}}, & 1 \leq l \leq k+1, \\
\frac{x_{l-3 k-3}\left(y_{l-3 k-3}+y_{l-2 k-2}\right)}{x_{l-3 k-3}-x_{l-2 k-2}}, & k+2 \leq l \leq 2 k+2, \\
\frac{x_{l-3 k-3}\left(y_{l-4 k-4}+y_{l-3 k-3}\right)}{-x_{l-4 k-4}+x_{l-3 k-3}}, & 2 k+3 \leq l \leq 3 k+3, \\
\frac{x_{l-4 k-4} y_{l-5 k-5}}{x_{l-5 k-5}-x_{l-4 k-4}}, & 3 k+4 \leq l \leq 4 k+4, \\
y_{l-6 k-6}, & 4 k+5 \leq l \leq 6 k+6,\end{cases}
\end{gathered}
$$

for $m \in \mathbb{N}_{0}$. 
Since equation (4) is a special case of equation (6), then similar to (12)-(15), we see that the system is equivalent to $k+1$ independent systems of the following form:

$$
\widehat{x}_{m+1}=\frac{\widehat{x}_{m} \widehat{y}_{m-1}}{\widehat{y}_{m-1}+\widehat{y}_{m}}, \quad \widehat{y}_{m+1}=\frac{\widehat{y}_{m} \widehat{x}_{m-1}}{-\widehat{x}_{m-1}+\widehat{x}_{m}}, \quad m \geq-1
$$

By using the change of variables

$$
\widehat{y}_{m}=(-1)^{m} \tilde{y}_{m}, \quad m \geq-2,
$$

in (43), it easily follows that

$$
\widehat{x}_{m+1}=\frac{\widehat{x}_{m} \tilde{y}_{m-1}}{\widetilde{y}_{m-1}-\tilde{y}_{m}}, \quad \tilde{y}_{m+1}=\frac{\tilde{y}_{m} \widehat{x}_{m-1}}{\widehat{x}_{m-1}-\widehat{x}_{m}}, \quad m \geq-1 .
$$

Using Theorem 4 in [29] we see that every well-defined solution to system (45) is sixperiodic, from which along with (44) it follows that every well-defined solution to system (43) is six-periodic. Hence, every well-defined solution to system (4) is $6(k+1)$-periodic. So, Theorem 3(i) is also a simple consequence of known results.

Part (ii) is a simple consequence of the independence of solutions $\left(x_{m}^{(j)}, y_{m}^{(j)}\right)_{m \geq-2}, j=$ $\overline{1, k+1}$, to system (45) and the following equalities for terms $x_{j}, y_{j}, j=\overline{0,5}$, where $\widehat{x}_{-2}$, $\widehat{y}_{-2}, \widehat{x}_{-1}$, and $\widehat{y}_{-1}$ are replaced with the initial values $x_{j-(2 k+2)}, y_{j-(2 k+2)}, x_{j-(k+1)}$, and $y_{j-(k+1)}$, $j=\overline{1, k+1}$, respectively. To get $\widehat{x}_{j}$ and $\widehat{y}_{j}, j=\overline{0,5}$, we can simply use (44) and (16)-(27). Namely, we have

$$
\begin{aligned}
& \widehat{x}_{0}=\frac{\widehat{x}_{-1} \tilde{y}_{-2}}{\widetilde{y}_{-2}-\widetilde{y}_{-1}}=\frac{\widehat{x}_{-1} \widehat{y}_{-2}}{\widehat{y}_{-2}+\widehat{y}_{-1}}, \\
& \widehat{x}_{1}=-\frac{\widetilde{y}_{-2}\left(\widehat{x}_{-2}-\widehat{x}_{-1}\right)}{\widetilde{y}_{-2}-\widetilde{y}_{-1}}=\frac{\widehat{y}_{-2}\left(-\widehat{x}_{-2}+\widehat{x}_{-1}\right)}{\widehat{y}_{-2}+\widehat{y}_{-1}}, \\
& \widehat{x}_{2}=-\frac{\widetilde{y}_{-1}\left(\widehat{x}_{-2}-\widehat{x}_{-1}\right)}{\widetilde{y}_{-2}-\widetilde{y}_{-1}}=\frac{\widehat{y}_{-1}\left(\widehat{x}_{-2}-\widehat{x}_{-1}\right)}{\widehat{y}_{-2}+\widehat{y}_{-1}}, \\
& \widehat{x}_{3}=-\frac{\widehat{x}_{-2} \tilde{y}_{-1}}{\widetilde{y}_{-2}-\widetilde{y}_{-1}}=\frac{\widehat{x}_{-2} \widehat{y}_{-1}}{\widehat{y}_{-2}+\widehat{y}_{-1}}, \\
& \widehat{x}_{4}=\widehat{x}_{-2}, \\
& \widehat{x}_{5}=\widehat{x}_{-1},
\end{aligned}
$$

and

$$
\begin{aligned}
& \widehat{y}_{0}=\tilde{y}_{0}=\frac{\tilde{y}_{-1} \widehat{x}_{-2}}{\widehat{x}_{-2}-\widehat{x}_{-1}}=-\frac{\widehat{y}_{-1} \widehat{x}_{-2}}{\widehat{x}_{-2}-\widehat{x}_{-1}}, \\
& \widehat{y}_{1}=-\widetilde{y}_{1}=\frac{\widehat{x}_{-2}\left(\widetilde{y}_{-2}-\tilde{y}_{-1}\right)}{\widehat{x}_{-2}-\widehat{x}_{-1}}=\frac{\widehat{x}_{-2}\left(\widehat{y}_{-2}+\widehat{y}_{-1}\right)}{\widehat{x}_{-2}-\widehat{x}_{-1}}, \\
& \widehat{y}_{2}=\widetilde{y}_{2}=-\frac{\widehat{x}_{-1}\left(\widetilde{y}_{-2}-\tilde{y}_{-1}\right)}{\widehat{x}_{-2}-\widehat{x}_{-1}}=\frac{\widehat{x}_{-1}\left(\widehat{y}_{-2}+\widehat{y}_{-1}\right)}{-\widehat{x}_{-2}+\widehat{x}_{-1}}, \\
& \widehat{y}_{3}=-\widetilde{y}_{3}=\frac{\widetilde{y}_{-2} \widehat{x}_{-1}}{\widehat{x}_{-2}-\widehat{x}_{-1}}=\frac{\widehat{y}_{-2} \widehat{x}_{-1}}{\widehat{x}_{-2}-\widehat{x}_{-1}},
\end{aligned}
$$




$$
\begin{aligned}
& \widehat{y}_{4}=\tilde{y}_{4}=\tilde{y}_{-2}=\widehat{y}_{-2}, \\
& \widehat{y}_{5}=-\tilde{y}_{5}=-\tilde{y}_{-1}=\widehat{y}_{-1} .
\end{aligned}
$$

Theorem 4 Let $\left(x_{n}, y_{n}\right)_{n \geq-(2 k+1)}$ be a well-defined solution to system (5). Then the following statements hold.

(i) Sequences $\left(x_{n}\right)_{n \geq-(2 k+1)}$ and $\left(y_{n}\right)_{n \geq-(2 k+1)}$ are periodic with period $6(k+1)$.

(ii) We have

$$
\begin{aligned}
& x_{6(k+1) m+l}= \begin{cases}\frac{x_{l-k-1} y_{l-2 k-2}}{-y_{l-2 k-2}+y_{l-k-1}}, & 1 \leq l \leq k+1, \\
\frac{y_{l-3 k-3}\left(x_{l-3 k-3}+x_{l-2 k-2}\right)}{y_{l-3 k-3}-y_{l-2 k-2}}, & k+2 \leq l \leq 2 k+2, \\
\frac{y_{l-3 k-3}\left(x_{l-4 k-4}+l_{l-3 k-3}\right)}{-y_{l-4 k-4}+y_{l-3 k-3}}, & 2 k+3 \leq l \leq 3 k+3, \\
\frac{y_{l-4 k-4} x_{l-5 k-5}}{y_{l-5 k-5}-y_{l-4 k-4}}, & 3 k+4 \leq l \leq 4 k+4, \\
x_{l-6 k-6}, & 4 k+5 \leq l \leq 6 k+6,\end{cases} \\
& y_{6(k+1) m+l}= \begin{cases}\frac{y_{l-k-1} x_{l-2 k-2}}{x_{l-2 k-2}+x_{l-k-1}}, & 1 \leq l \leq k+1, \\
\frac{x_{l-3 k-3}\left(-y_{l-3 k-3}+y_{l-2 k-2}\right)}{x_{l-3 k-3}+x_{l-2 k-2}}, & k+2 \leq l \leq 2 k+2, \\
\frac{x_{l-3 k-3}\left(y_{l-4 k-4}-y_{l-3 k-3}\right)}{x_{-1 k-4+x}+x_{l-3 k-3}}, & 2 k+3 \leq l \leq 3 k+3, \\
\frac{x_{l-4 k-4} y_{l-5 k-5},}{x_{l-5 k-5}+x_{l-4 k-4}}, & 3 k+4 \leq l \leq 4 k+4, \\
y_{l-6 k-6}, & 4 k+5 \leq l \leq 6 k+6,\end{cases}
\end{aligned}
$$

for $m \in \mathbb{N}_{0}$.

Since system (5) is also a special case of system (6), we see that the system is equivalent to $k+1$ independent systems of the following form:

$$
\widehat{x}_{m+1}=\frac{\widehat{x}_{m} \widehat{y}_{m-1}}{-\widehat{y}_{m-1}+\widehat{y}_{m}}, \quad \widehat{y}_{m+1}=\frac{\widehat{y}_{m} \widehat{x}_{m-1}}{\widehat{x}_{m-1}+\widehat{x}_{m}}, \quad m \geq-1
$$

Note that equation (58) is obtained from equation (43) by interchanging letters $x$ and $y$. Thus, Theorem 4 directly follows from Theorem 3 , which as we have already mentioned follows from known results, so it is also the case with Theorem 4.

Remark 1 By using the change of variables

$$
\widehat{x}_{m}=(-1)^{m} \widetilde{x}_{m}, \quad m \geq-2,
$$

in (58), it easily follows that

$$
\widetilde{x}_{m+1}=\frac{\widetilde{x}_{m} \widehat{y}_{m-1}}{\widehat{y}_{m-1}-\widehat{y}_{m}}, \quad \widehat{y}_{m+1}=\frac{\widehat{y}_{m} \widetilde{x}_{m-1}}{\widetilde{x}_{m-1}-\widetilde{x}_{m}}, \quad m \geq-1
$$

Hence, Theorem 4 can be also proved by Theorem 4 in [29], as it was the case with Theorems 2 and 3.

Remark 2 Note that the changes of variables (29) in Theorem 2, (44) in Theorem 3, (59) in Remark 1, along with the interlacing argument explained above show that systems (3)-(5) 
are equivalent to system (2), so essentially only one result was proved in [46], which as we have explained follows from the known ones.

\section{Solvability of some extensions of system (6)}

Here we show how some extensions of system (6) can be solved.

\subsection{A three-dimensional extension to system (6)}

The following three-dimensional system of difference equations is a natural generalization of two-dimensional system (6):

$$
\begin{aligned}
x_{n+1} & =\frac{x_{n-k} y_{n-(2 k+1)}}{a y_{n-(2 k+1)}+b y_{n-k}}, \\
y_{n+1} & =\frac{y_{n-k} z_{n-(2 k+1)}}{c z_{n-(2 k+1)}+d z_{n-k}}, \\
z_{n+1} & =\frac{z_{n-k} x_{n-(2 k+1)}}{e x_{n-(2 k+1)}+f x_{n-k}},
\end{aligned}
$$

for $n \in \mathbb{N}_{0}$, where parameters $a, b, c, d, e, f$ and initial values $x_{-j}, y_{-j}, z_{-j}, j=\overline{0,2 k+1}$, are complex numbers, and $k \in \mathbb{N}_{0}$.

As in the previous section, it is proved that system (61) is a system of difference equations with interlacing indices of order $k+1$.

Let

$$
\begin{aligned}
& x_{m}^{(j)}:=x_{(k+1) m+j}, \\
& y_{m}^{(j)}:=y_{(k+1) m+j}, \\
& z_{m}^{(j)}:=z_{(k+1) m+j},
\end{aligned}
$$

for $m \geq-2$, where $j=\overline{1, k+1}$.

Employing (62)-(64) in (61), we see that the system can be written as

$$
\begin{aligned}
& x_{m+1}^{(j)}=\frac{x_{m}^{(j)} y_{m-1}^{(j)}}{a y_{m-1}^{(j)}+b y_{m}^{(j)}}, \\
& y_{m+1}^{(j)}=\frac{y_{m}^{(j)} z_{m-1}^{(j)}}{c z_{m-1}^{(j)}+d z_{m}^{(j)}}, \\
& z_{m+1}^{(j)}=\frac{z_{m}^{(j)} x_{m-1}^{(j)}}{e x_{m-1}^{(j)}+f x_{m}^{(j)}},
\end{aligned}
$$

for $m \geq-1$ and $j=\overline{1, k+1}$.

From this it follows that the sequences $\left(x_{m}^{(j)}, y_{m}^{(j)}, z_{m}^{(j)}\right)_{m \geq-2}, j=\overline{1, k+1}$, are $k+1$ independent solutions to the following second-order system of difference equations:

$$
\begin{aligned}
& \widehat{x}_{m+1}=\frac{\widehat{x}_{m} \widehat{y}_{m-1}}{a \widehat{y}_{m-1}+b \widehat{y}_{m}}, \\
& \widehat{y}_{m+1}=\frac{\widehat{y}_{m} \widehat{z}_{m-1}}{c \widehat{z}_{m-1}+d \widehat{z}_{m}}, \\
& \widehat{z}_{m+1}=\frac{\widehat{z}_{m} \widehat{x}_{m-1}}{e \widehat{x}_{m-1}+f \widehat{x}_{m}},
\end{aligned}
$$


for $m \geq-1$, with the initial values $x_{j-(2 k+2)}, y_{j-(2 k+2)}, z_{j-(2 k+2)}, x_{j-(k+1)}, y_{j-(k+1)}$, and $z_{j-(k+1)}$, $j=\overline{1, k+1}$, respectively.

By using the changes of variables

$$
u_{m}=\frac{\widehat{x}_{m}}{\widehat{x}_{m-1}}, \quad v_{m}=\frac{\widehat{y}_{m}}{\widehat{y}_{m-1}}, \quad w_{n}=\frac{\widehat{z}_{m}}{\widehat{z}_{m-1}},
$$

$m \geq-1$, system (65) can be written in the following form:

$$
u_{m+1}=\frac{1}{a+b v_{m}}, \quad v_{m+1}=\frac{1}{c+d w_{m}}, \quad w_{m+1}=\frac{1}{e+f u_{m}},
$$

for $m \geq-1$.

From (67) we obtain

$$
\begin{aligned}
u_{m+1} & =\frac{1}{a+b v_{m}}=\frac{1}{a+\frac{b}{c+d w_{m-1}}}=\frac{c+d w_{m-1}}{a c+b+a d w_{m-1}}=\frac{c+\frac{d}{e+f u_{m-2}}}{a c+b+\frac{a d}{e+f u_{m-2}}} \\
& =\frac{c e+d+c f u_{m-2}}{a c e+b e+a d+(a c f+b f) u_{m-2}}, \\
v_{m+1} & =\frac{1}{c+d w_{m}}=\frac{1}{c+\frac{d}{e+f u_{m-1}}}=\frac{e+f u_{m-1}}{c e+d+c f u_{m-1}}=\frac{e+\frac{f}{a+b v_{m-2}}}{c e+d+\frac{c f}{a+b v_{m-2}}} \\
& =\frac{a e+f+b e v_{m-2}}{a c e+a d+c f+(b c e+b d) v_{m-2}}, \\
w_{m+1} & =\frac{1}{e+f u_{m}}=\frac{1}{e+\frac{f}{a+b v_{m-1}}}=\frac{a+b v_{m-1}}{a e+f+b e v_{m-1}}=\frac{a+\frac{b}{c+d w_{m-2}}}{a e+f+\frac{b e}{c+d w_{m-2}}} \\
& =\frac{a c+b+a d w_{m-2}}{a c e+b e+c f+(a d e+d f) w_{m-2}},
\end{aligned}
$$

for $m \in \mathbb{N}$.

Using the procedure in Sect. 2, it is seen that equations (68)-(70) are three equations with interlacing indices of order three.

Let

$$
\begin{aligned}
& u_{k}^{(j)}:=u_{3 k+j}, \\
& v_{k}^{(j)}:=v_{3 k+j}, \\
& w_{k}^{(j)}:=w_{3 k+j},
\end{aligned}
$$

for $k \in \mathbb{N}_{0}$, where $j \in\{-1,0,1\}$.

Employing (71)-(73) in (68)-(70), respectively, we see that the equations can be written as

$$
\begin{aligned}
& u_{k+1}^{(j)}=\frac{c e+d+c f u_{k}^{(j)}}{a c e+b e+a d+(a c f+b f) u_{k}^{(j)}}, \\
& v_{k+1}^{(j)}=\frac{a e+f+b e v_{k}^{(j)}}{a c e+a d+c f+(b c e+b d) v_{k}^{(j)}},
\end{aligned}
$$




$$
w_{k+1}^{(j)}=\frac{a c+b+a d w_{k}^{(j)}}{a c e+b e+c f+(a d e+d f) w_{k}^{(j)}},
$$

for $k \in \mathbb{N}_{0}$ and for each $j \in\{-1,0,1\}$.

From this it follows that the sequences $\left(u_{k}^{(j)}\right)_{k \in \mathbb{N}_{0}},\left(v_{k}^{(j)}\right)_{k \in \mathbb{N}_{0}},\left(w_{k}^{(j)}\right)_{k \in \mathbb{N}_{0}}, j \in\{-1,0,1\}$, are three solutions to the following bilinear difference equations:

$$
\begin{aligned}
& u_{k+1}=\frac{c e+d+c f u_{k}}{a c e+b e+a d+(a c f+b f) u_{k}}, \\
& v_{k+1}=\frac{a e+f+b e v_{k}}{a c e+a d+c f+(b c e+b d) v_{k}}, \\
& w_{k+1}=\frac{a c+b+a d w_{k}}{a c e+b e+c f+(a d e+d f) w_{k}},
\end{aligned}
$$

for $k \in \mathbb{N}_{0}$, and with the initial values $u_{j}, v_{j}, w_{j}, j \in\{-1,0,1\}$, respectively.

Since bilinear difference equations are solvable, it follows that systems (77)-(79) are also solvable. From this and (71)-(76) it follows that equations (68)-(70) are solvable, from which along with (66) it easily follows that system (61) is also solvable.

\subsection{A four-dimensional extension to system (6)}

The following four-dimensional system is another generalization of two-dimensional system (6):

$$
\begin{aligned}
& x_{n+1}=\frac{x_{n-k} y_{n-(2 k+1)}}{a y_{n-(2 k+1)}+b y_{n-k}}, \\
& y_{n+1}=\frac{y_{n-k} z_{n-(2 k+1)}}{c z_{n-(2 k+1)}+d z_{n-k}}, \\
& z_{n+1}=\frac{z_{n-k} u_{n-(2 k+1)}}{e u_{n-(2 k+1)}+f u_{n-k}}, \\
& u_{n+1}=\frac{u_{n-k} x_{n-(2 k+1)}}{g x_{n-(2 k+1)}+h x_{n-k}},
\end{aligned}
$$

for $n \in \mathbb{N}_{0}$, where parameters $a, b, c, d, e, f, g, h$ and initial values $x_{-j}, y_{-j}, z_{-j}, u_{-j}, j=$ $\overline{0,2 k+1}$, are complex numbers, and $k \in \mathbb{N}_{0}$.

As in Sect. 2 it is proved that system (80) is a system of difference equations with interlacing indices of order $k+1$.

Let

$$
\begin{aligned}
& x_{m}^{(j)}:=x_{(k+1) m+j}, \\
& y_{m}^{(j)}:=y_{(k+1) m+j}, \\
& z_{m}^{(j)}:=z_{(k+1) m+j}, \\
& u_{m}^{(j)}:=u_{(k+1) m+j},
\end{aligned}
$$

for $m \geq-2$, and $j=\overline{1, k+1}$.

Employing (81)-(84) in (80), we see that the system can be written as

$$
x_{m+1}^{(j)}=\frac{x_{m}^{(j)} y_{m-1}^{(j)}}{a y_{m-1}^{(j)}+b y_{m}^{(j)}},
$$




$$
\begin{aligned}
& y_{m+1}^{(j)}=\frac{y_{m}^{(j)} z_{m-1}^{(j)}}{c z_{m-1}^{(j)}+d z_{m}^{(j)}}, \\
& z_{m+1}^{(j)}=\frac{z_{m}^{(j)} u_{m-1}^{(j)}}{e u_{m-1}^{(j)}+f u_{m}^{(j)}}, \\
& u_{m+1}^{(j)}=\frac{u_{m}^{(j)} x_{m-1}^{(j)}}{g x_{m-1}^{(j)}+h x_{m}^{(j)}},
\end{aligned}
$$

for $m \geq-1$ and $j=\overline{1, k+1}$.

From this it follows that the sequences $\left(x_{m}^{(j)}, y_{m}^{(j)}, z_{m}^{(j)}, u_{m}^{(j)}\right)_{m \geq-2}, j=\overline{1, k+1}$, are $k+1$ independent solutions to the following second-order system of difference equations:

$$
\begin{aligned}
& \widehat{x}_{m+1}=\frac{\widehat{x}_{m} \widehat{y}_{m-1}}{a \widehat{y y}_{m-1}+b \widehat{y}_{m}}, \\
& \widehat{y}_{m+1}=\frac{\widehat{y}_{m} \widehat{z}_{m-1}}{c \widehat{z}_{m-1}+d \widehat{z}_{m}}, \\
& \widehat{z}_{m+1}=\frac{\widehat{z}_{m} \widehat{u}_{m-1}}{e \widehat{u}_{m-1}+f \widehat{u}_{m}}, \\
& \widehat{u}_{m+1}=\frac{\widehat{u}_{m} \widehat{x}_{m-1}}{g \widehat{x}_{m-1}+h \widehat{x}_{m}},
\end{aligned}
$$

for $m \geq-1$, with the initial values $x_{j-(2 k+2)}, y_{j-(2 k+2)}, z_{j-(2 k+2)}, u_{j-(2 k+2)}, x_{j-(k+1)}, y_{j-(k+1)}, z_{j-(k+1)}$, and $u_{j-(k+1)}, j=\overline{1, k+1}$, respectively.

By using the changes of variables

$$
\alpha_{m}=\frac{\widehat{x}_{m}}{\widehat{x}_{m-1}}, \quad \beta_{m}=\frac{\widehat{y}_{m}}{\widehat{y}_{m-1}}, \quad \gamma_{m}=\frac{\widehat{z}_{m}}{\widehat{z}_{m-1}}, \quad \delta_{m}=\frac{\widehat{u}_{m}}{\widehat{u}_{m-1}}
$$

$m \geq-1$, system (85) can be written in the following form:

$$
\begin{aligned}
& \alpha_{m+1}=\frac{1}{a+b \beta_{m}}, \\
& \beta_{m+1}=\frac{1}{c+d \gamma_{m}}, \\
& \gamma_{m+1}=\frac{1}{e+f \delta_{m}}, \\
& \delta_{m+1}=\frac{1}{g+h \alpha_{m}},
\end{aligned}
$$

for $m \geq-1$.

From (87) we obtain

$$
\begin{aligned}
\alpha_{m+1} & =\frac{1}{a+b \beta_{m}}=\frac{1}{a+\frac{b}{c+d \gamma_{m-1}}}=\frac{c+d \gamma_{m-1}}{a c+b+a d \gamma_{m-1}}=\frac{c+\frac{d}{e+f \delta_{m-2}}}{a c+b+\frac{a d}{e+f \delta_{m-2}}} \\
& =\frac{c e+d+c f \delta_{m-2}}{a c e+b e+a d+(a c f+b f) \delta_{m-2}}
\end{aligned}
$$




$$
\begin{aligned}
& =\frac{c e+d+\frac{c f}{g+h \alpha_{m-3}}}{a c e+b e+a d+\frac{a c f+b f}{g+h \alpha_{m-3}}} \\
& =\frac{c e g+d g+c f+(c e h+d h) \alpha_{m-3}}{a c e g+b e g+a d g+a c f+b f+(a c e h+b e h+a d h) \alpha_{m-3}}, \\
& \beta_{m+1}=\frac{1}{c+d \gamma_{m}}=\frac{1}{c+\frac{d}{e+f \delta_{m-1}}}=\frac{e+f \delta_{m-1}}{c e+d+c f \delta_{m-1}}=\frac{e+\frac{f}{g+h \alpha_{m-2}}}{c e+d+\frac{c f}{g+h \alpha_{m-2}}} \\
& =\frac{e g+f+e h \alpha_{m-2}}{c e g+d g+c f+(c e h+d h) \alpha_{m-2}} \\
& =\frac{e g+f+\frac{e h}{a+b \beta_{m-3}}}{c e g+d g+c f+\frac{c e h+d h}{a+b \beta_{m-3}}} \\
& =\frac{a e g+a f+e h+(b e g+b f) \beta_{m-3}}{a c e g+a d g+a c f+c e h+d h+(b c e g+b d g+b c f) \beta_{m-3}}, \\
& \gamma_{m+1}=\frac{1}{e+f \delta_{m}}=\frac{1}{e+\frac{f}{g+h \alpha_{m-1}}}=\frac{g+h \alpha_{m-1}}{e g+f+e h \alpha_{m-1}}=\frac{g+\frac{h}{a+b \beta_{m-2}}}{e g+f+\frac{e h}{a+b \beta_{m-2}}} \\
& =\frac{a g+h+b g \beta_{m-2}}{a e g+a f+e h+(b e g+b f) \beta_{m-2}} \\
& =\frac{a g+h+\frac{b g}{c+d \gamma_{m-3}}}{a e g+a f+e h+\frac{b e g+b f}{c+d \gamma_{m-3}}} \\
& =\frac{a c g+c h+b g+(a d g+d h) \gamma_{m-3}}{a c e g+a c f+c e h+b e g+b f+(a d e g+a d f+d e h) \gamma_{m-3}}, \\
& \delta_{m+1}=\frac{1}{g+h \alpha_{m}}=\frac{1}{g+\frac{h}{a+b \beta_{m-1}}}=\frac{a+b \beta_{m-1}}{a g+h+b g \beta_{m-1}}=\frac{a+\frac{b}{c+d \gamma_{m-2}}}{a g+h+\frac{b g}{c+d \gamma_{m-2}}} \\
& =\frac{a c+b+a d \gamma_{m-2}}{a c g+b g+c h+(a d g+d h) \gamma_{m-2}} \\
& =\frac{a c+b+\frac{a d}{e+f \delta_{m-3}}}{a c g+b g+c h+\frac{a d g+d h}{e+f \delta_{m-3}}} \\
& =\frac{a c e+b e+a d+(a c f+b f) \delta_{m-3}}{a c e g+b e g+c e h+a d g+d h+(a c f g+b f g+c f h) \delta_{m-3}},
\end{aligned}
$$

for $m \geq 2$.

Using the procedure from Sect. 2, it is seen that equations (88)-(91) are four equations with interlacing indices of order four.

Let

$$
\begin{aligned}
& \alpha_{k}^{(j)}:=\alpha_{4 k+j}, \\
& \beta_{k}^{(j)}:=\beta_{4 k+j}, \\
& \gamma_{k}^{(j)}:=\gamma_{4 k+j}, \\
& \delta_{k}^{(j)}:=\delta_{4 k+j},
\end{aligned}
$$

for $k \in \mathbb{N}_{0}$, where $j \in\{-1,0,1,2\}$. 
Employing (92)-(95) in (88)-(91), respectively, we see that the equations can be written as

$$
\begin{aligned}
& \alpha_{k+1}^{(j)}=\frac{c e g+d g+c f+(c e h+d h) \alpha_{k}^{(j)}}{a c e g+b e g+a d g+a c f+b f+(a c e h+b e h+a d h) \alpha_{k}^{(j)}}, \\
& \beta_{k+1}^{(j)}=\frac{a e g+a f+e h+(b e g+b f) \beta_{k}^{(j)}}{a c e g+a d g+a c f+c e h+d h+(b c e g+b d g+b c f) \beta_{k}^{(j)}}, \\
& \gamma_{k+1}^{(j)}=\frac{a c g+c h+b g+(a d g+d h) \gamma_{k}^{(j)}}{a c e g+a c f+c e h+b e g+b f+(a d e g+a d f+d e h) \gamma_{k}^{(j)}}, \\
& \delta_{k+1}^{(j)}=\frac{a c e+b e+a d+(a c f+b f) \delta_{k}^{(j)}}{a c e g+b e g+c e h+a d g+d h+(a c f g+b f g+c f h) \delta_{k}^{(j)}},
\end{aligned}
$$

for $k \in \mathbb{N}_{0}$ and for each $j \in\{-1,0,1,2\}$.

From this it follows that the sequences $\left(\alpha_{k}^{(j)}\right)_{k \in \mathbb{N}_{0}},\left(\beta_{k}^{(j)}\right)_{k \in \mathbb{N}_{0}},\left(\gamma_{k}^{(j)}\right)_{k \in \mathbb{N}_{0}},\left(\delta_{k}^{(j)}\right)_{k \in \mathbb{N}_{0}}, j \in$ $\{-1,0,1,2\}$, are four solutions to the following bilinear difference equations:

$$
\begin{aligned}
& \widehat{\alpha}_{k+1}=\frac{c e g+d g+c f+(c e h+d h) \widehat{\alpha}_{k}}{a c e g+b e g+a d g+a c f+b f+(a c e h+b e h+a d h) \widehat{\alpha}_{k}}, \\
& \widehat{\beta}_{k+1}=\frac{a e g+a f+e h+(b e g+b f) \widehat{\beta}_{k}}{a c e g+a d g+a c f+c e h+d h+(b c e g+b d g+b c f) \widehat{\beta}_{k}}, \\
& \widehat{\gamma}_{k+1}=\frac{a c g+c h+b g+(a d g+d h) \widehat{\gamma}_{k}}{a c e g+a c f+c e h+b e g+b f+(a d e g+a d f+d e h) \widehat{\gamma}_{k}}, \\
& \widehat{\delta}_{k+1}=\frac{a c e+b e+a d+(a c f+b f) \widehat{\delta}_{k}}{a c e g+b e g+c e h+a d g+d h+(a c f g+b f g+c f h) \widehat{\delta}_{k}},
\end{aligned}
$$

for $k \in \mathbb{N}_{0}$, with the initial values $\alpha_{j}, \beta_{j}, \gamma_{j}, \delta_{j}, j \in\{-1,0,1,2\}$, respectively.

Since bilinear difference equations are solvable in closed form, it follows that systems (100)-(103) are also solvable. From this along with (92)-(99) it follows that equations (88)-(91) are solvable, from which and (86) it follows that system (80) is also solvable.

Remark 3 We will not present closed-form formulas for solutions to systems (61) and (80) since it is done in a standard way by using a method for solving bilinear difference equations along with the changes of variables given in this section. By using such obtained closed-form formulas for solutions to the systems, the long-term behavior of their solutions can be described, which can be done as in our papers [28, 29, 34]. We leave the standard problem to the reader as an exercise.

Remark 4 The corresponding extensions of system (2), which are obtained from systems (61) and (80), are the following:

$$
\begin{aligned}
& x_{n+1}=\frac{x_{n-k} y_{n-(2 k+1)}}{y_{n-(2 k+1)}-y_{n-k}}, \\
& y_{n+1}=\frac{y_{n-k} z_{n-(2 k+1)}}{z_{n-(2 k+1)}-z_{n-k}}, \\
& z_{n+1}=\frac{z_{n-k} x_{n-(2 k+1)}}{x_{n-(2 k+1)}-x_{n-k}},
\end{aligned}
$$


for $n \in \mathbb{N}_{0}$, where $k \in \mathbb{N}_{0}$, and

$$
\begin{aligned}
& x_{n+1}=\frac{x_{n-k} y_{n-(2 k+1)}}{y_{n-(2 k+1)}-y_{n-k}}, \\
& y_{n+1}=\frac{y_{n-k} z_{n-(2 k+1)}}{z_{n-(2 k+1)}-z_{n-k}}, \\
& z_{n+1}=\frac{z_{n-k} u_{n-(2 k+1)}}{u_{n-(2 k+1)}-u_{n-k}}, \\
& u_{n+1}=\frac{u_{n-k} x_{n-(2 k+1)}}{x_{n-(2 k+1)}-x_{n-k}},
\end{aligned}
$$

for $n \in \mathbb{N}_{0}$, where $k \in \mathbb{N}_{0}$.

Since systems (104) and (105) are three- and four-dimensional cyclic systems, respectively, and the scalar difference equation

$$
x_{n+1}=\frac{x_{n-k} x_{n-(2 k+1)}}{x_{n-(2 k+1)}-x_{n-k}}
$$

is periodic with period $6(k+1)$ (as an interlacing equation of order $k+1$ and since for $k=0$ an equation of period six is obtained; see also equalities (16)-(21), where letter $y$ is replaced by letter $x$ ), it is known that system (104) must be periodic with period $18(k+1)$, whereas (105) must be periodic with period $24(k+1)$.

\section{Acknowledgements}

The work of Bratislav Iričanin was supported by the Serbian Ministry of Education and Science projects III 41025 and OI 171007, work of Stevo Stević by projects III 41025 and III 44006, while the work of Zdeněk Šmarda was supported by the project FEKT-S-17-4225 of Brno University of Technology.

\section{Funding}

FEKT-S-17-4225 of Brno University of Technology.

Availability of data and materials

Not applicable.

\section{Competing interests}

The authors declare that they have no competing interests.

\section{Authors' contributions}

All authors contributed equally to the writing of this paper. All authors read and approved the manuscript.

\section{Author details}

${ }^{1}$ Mathematical Institute of the Serbian Academy of Sciences, Beograd, Serbia. ${ }^{2}$ Department of Medical Research, China Medical University Hospital, China Medical University, Taichung, Taiwan, Republic of China. ${ }^{3}$ Department of Computer Science and Information Engineering, Asia University, Taichung, Taiwan, Republic of China. ${ }^{4}$ Department of Mathematics, Faculty of Electrical Engineering and Communication, Brno University of Technology, Brno, Czech Republic. ${ }^{5}$ Faculty of Electrical Engineering, Belgrade University, Beograd, Serbia. ${ }^{6}$ Department of Mathematical Sciences, Appalachian State University, Boone, USA.

\section{Publisher's Note}

Springer Nature remains neutral with regard to jurisdictional claims in published maps and institutional affiliations.

Received: 22 November 2018 Accepted: 15 January 2019 Published online: 29 January 2019

\section{References}

1. Agarwal, R.P.: Difference Equations and Inequalities: Theory, Methods, and Applications, 2nd edn. Dekker, New York (2000)

2. Boole, G.: A Treatsie on the Calculus of Finite Differences, 3rd edn. Macmillan \& Co., London (1880)

3. Fort, T.: Finite Differences and Difference Equations in the Real Domain. Clarendion Press, Oxford (1948)

4. Jordan, C.: Calculus of Finite Differences. Chelsea, New York (1956)

5. Krechmar, V.A.: A Problem Book in Algebra. Mir, Moscow (1974) (Russian first edition 1937) 
6. Markoff, A.A.: Differenzenrechnung, Leipzig (1896) (in German)

7. Milne-Thomson, L.M.: The Calculus of Finite Differences. MacMillan \& Co., London (1933)

8. Mitrinović, D.S., Kečkić, J.D.: Methods for Calculating Finite Sums. Naučna Knjiga, Beograd (1984) (in Serbian)

9. Nörlund, N.E.: Vorlesungen über Differenzenrechnung. Springer, Berlin (1924) (in German)

10. Richardson, C.H.: An Introduction to the Calculus of Finite Differences. Van Nostrand, Toronto (1954)

11. Stević, S.: Bounded solutions to nonhomogeneous linear second-order difference equations. Symmetry 9, Article ID $227(2017)$

12. Agarwal, R.P., Popenda, J.: Periodic solutions of first order linear difference equations. Math. Comput. Model. 22(1), 11-19 (1995)

13. Berezansky, L., Braverman, E.: On impulsive Beverton-Holt difference equations and their applications. J. Differ. Equ. Appl. 10(9), 851-868 (2004)

14. Mitrinović, D.S.: Matrices and Determinants. Naučna Knjiga, Beograd (1989) (in Serbian)

15. Mitrinović, D.S., Adamović, D.D.: Sequences and Series. Naučna Knjiga, Beograd (1980) (in Serbian)

16. Riordan, J.: Combinatorial Identities. Wiley, New York (1968)

17. Stević, S.: First-order product-type systems of difference equations solvable in closed form. Electron. J. Differ. Equ. 2015, Article ID 308 (2015)

18. Stević, S.: Existence of a unique bounded solution to a linear second order difference equation and the linear first order difference equation. Adv. Differ. Equ. 2017, Article ID 169 (2017)

19. Stević, S., Alghamdi, M.A., Alotaibi, A., Elsayed, E.M.: Solvable product-type system of difference equations of second order. Electron. J. Differ. Equ. 2015, Article ID 169 (2015)

20. Brand, L.: Differential and Difference Equations. Wiley, New York (1966)

21. Adamović, D.: Solution to problem 194. Mat. Vesn. 23, 236-242 (1971)

22. Brand, L:: A sequence defined by a difference equation. Am. Math. Mon. 62(7), 489-492 (1955)

23. Stević, S.: Representation of solutions of bilinear difference equations in terms of generalized Fibonacci sequences. Electron. J. Qual. Theory Differ. Equ. 2014, Article ID 67 (2014)

24. Stević, S., Iričanin, B., Šmarda, Z.: On a symmetric bilinear system of difference equations. Appl. Math. Lett. 89, 15-21 (2019)

25. Alfred, B.U:: An Introduction to Fibonacci Discovery, the Fibonacci Association (1965)

26. Vorobiev, N.N.: Fibonacci Numbers. Birkhäuser, Basel (2002) (Russian original 1950)

27. Stević, S., Diblik, J., Iričanin, B., Šmarda, Z.: On some solvable difference equations and systems of difference equations. Abstr. Appl. Anal. 2012, Article ID 541761 (2012)

28. Stević, S., Diblik, J., Iričanin, B., Šmarda, Z.: Solvability of nonlinear difference equations of fourth order. Electron. J. Differ. Equ. 2014, Article ID 264 (2014)

29. Stević, S., Diblik, J., Iričanin, B., Šmarda, Z.: On the system of difference equations $x_{n}=x_{n-1} y_{n-2} /\left(a y_{n-2}+b y_{n-1}\right)$, $y_{n}=y_{n-1} x_{n-2} /\left(c x_{n-2}+d x_{n-1}\right)$. Appl. Math. Comput. 270, 688-704 (2015)

30. Papaschinopoulos, G., Stefanidou, G.: Asymptotic behavior of the solutions of a class of rational difference equations. Int. J. Difference Equ. 5(2), 233-249 (2010)

31. Stević, S.: On the difference equation $x_{n}=x_{n-2} /\left(b_{n}+c_{n} x_{n-1} x_{n-2}\right)$. Appl. Math. Comput. 218, 4507-4513 (2011)

32. Stević, S.: On the system of difference equations $x_{n}=c_{n} y_{n-3} /\left(a_{n}+b_{n} y_{n-1} x_{n-2} y_{n-3}\right), y_{n}=\gamma_{n} x_{n-3} /\left(\alpha_{n}+\beta_{n} x_{n-1} y_{n-2} x_{n-3}\right)$. Appl. Math. Comput. 219, 4755-4764 (2013)

33. Stević, S., Iričanin, B., Šmarda, Z.: Solvability of a close to symmetric system of difference equations. Electron. J. Differ Equ. 2016, Article ID 159 (2016)

34. Stević, S., Diblik, J., Iričanin, B., Šmarda, Z:: On a third-order system of difference equations with variable coefficients. Abstr. Appl. Anal. 2012, Article ID 508523 (2012)

35. Stević, S., Diblik, J., Iričanin, B., Šmarda, Z.: On a solvable system of rational difference equations. J. Differ. Equ. Appl. 20(5-6), 811-825 (2014)

36. Berg, L., Stević, S.: On some systems of difference equations. Appl. Math. Comput. 218, 1713-1718 (2011)

37. Papaschinopoulos, G., Schinas, C.J.: On a system of two nonlinear difference equations. J. Math. Anal. Appl. 219(2), 415-426 (1998)

38. Papaschinopoulos, G., Schinas, C.J.: On the behavior of the solutions of a system of two nonlinear difference equations. Commun. Appl. Nonlinear Anal. 5(2), 47-59 (1998)

39. Papaschinopoulos, G., Schinas, C.J.: Invariants for systems of two nonlinear difference equations. Differ. Equ. Dyn. Syst. 7, 181-196 (1999)

40. Papaschinopoulos, G., Schinas, C.J.: Invariants and oscillation for systems of two nonlinear difference equations. Nonlinear Anal., Theory Methods Appl. 46, 967-978 (2001)

41. Papaschinopoulos, G., Schinas, C.J.: Oscillation and asymptotic stability of two systems of difference equations of rational form. J. Differ. Equ. Appl. 7, 601-617 (2001)

42. Papaschinopoulos, G., Schinas, C.J.: On the dynamics of two exponential type systems of difference equations. Comput. Math. Appl. 64(7), 2326-2334 (2012)

43. Papaschinopoulos, G., Schinas, C.J., Stefanidou, G.: On a k-order system of Lyness-type difference equations. Adv. Differ. Equ. 2007, Article ID 31272 (2007)

44. Stefanidou, G., Papaschinopoulos, G., Schinas, C.: On a system of max difference equations. Dyn. Contin. Discrete Impuls. Syst., Ser. A 14(6), 885-903 (2007)

45. Andruch-Sobilo, A., Migda, M.: Further properties of the rational recursive sequence $x_{n+1}=a x_{n-1} /\left(b+c x_{n} x_{n-1}\right)$. Opusc. Math. 26(3), 387-394 (2006)

46. Gocen, M., Cebeci, A.: On the periodic solutions of some systems of higher order difference equations. Rocky Mt. J. Math. 48(3), 845-858 (2018)

47. Stević, S.: Bounded and periodic solutions to the linear first-order difference equation on the integer domain. Adv. Differ. Equ. 2017, Article ID 283 (2017)

48. Stević, S.: New class of solvable systems of difference equations. Appl. Math. Lett. 63, 137-144 (2017)

49. Stević, S.: Solvable product-type system of difference equations whose associated polynomial is of the fourth order. Electron. J. Qual. Theory Differ. Equ. 2017, Article ID 13 (2017) 
50. Stević, S., Iričanin, B., Šmarda, Z:: On a product-type system of difference equations of second order solvable in closed form. J. Inequal. Appl. 2015, Article ID 327 (2015)

51. Stević, S., Iričanin, B., Šmarda, Z: Two-dimensional product-type system of difference equations solvable in closed form. Adv. Differ. Equ. 2016, Article ID 253 (2016)

52. Stević, S.: Periodicity of max difference equations. Util. Math. 83, 69-71 (2010)

53. Stević, S.: On some periodic systems of max-type difference equations. Appl. Math. Comput. 218, 11483-11487 (2012)

54. Iričanin, B., Stević, S.: Eventually constant solutions of a rational difference equation. Appl. Math. Comput. 215 854-856 (2009)

Submit your manuscript to a SpringerOpen ${ }^{\circ}$ journal and benefit from:

- Convenient online submission

- Rigorous peer review

- Open access: articles freely available online

- High visibility within the field

- Retaining the copyright to your article

Submit your next manuscript at $\boldsymbol{~ s p r i n g e r o p e n . c o m ~}$ 